\title{
24
}

\section{Reliability of Laminated Structures by an Energy Based Failure Criterion}

\author{
S. Plica, R. Rackwitz, Technische Universität München, 80290 München, Germany
}

\begin{abstract}
Studies about the reliability of fibre reinforced laminated structures have mostly concentrated on single layers with simple failure criteria such as the well known by Tsai/Wu. In laminated structures failure frequently is defined as interfibre failure. A realistic criterion taking account of the multi-axial stress states has recently been proposed by Hashin and Puck. This criterion is briefly discussed and related to energy considerations. The criterion then is used in the analysis of a realistic tube structure whose laminate stresses are determined by classical laminate theory. Reliabilities are determined by FORM methods. Correlations among the various failure modes and between the elements of the FEM-model for the tube are taken into account. The size effect is studied with particular reference to the meshing.
\end{abstract}

\section{Introduction}

The properties of fibre reinforced plastics (FRP), i. e. high strength combined with low weight, together with the possibility to design materials with different elasticity and strength properties in different directions have led to an increased use, especially in the air- and spacecraft industry. Unfortunately a special problem when designing a laminate is that the fracture behaviour of laminates is not yet fully understood. Theoretical predictions and experimental results still can differ substantially, although much research work has been done on this topic during the last decades.

While the inhomogenity of laminates can be circumvented in a fracture analysis by simply calculating the stresses in the individual layers, the strength of a lamina poses more complex problems. Due to their orthotropy the unidirectional layers which are used to build up a laminate show extremly different strengths in fibre direction and in transverse direction. Two major failure modes can be identified, the failure of the fibres (FF) under tensile or compressive load and the failure of the matrix material between the fibres caused by transverse stresses or shear stresses. Although the failure of the matrix material shows several possible modes like tensile, compressive, longitudinal or transverse shear failure, all these are regarded as one mode, the interfibre failure (IFF).

In case of failure a fracture criterion should indicate whether FF or IFF has taken place. For practical purposes this information is important as a structure may be designed for fibreparallel strength, whence IFF does not affect its usability. However, for some structures IFF does not only cause unserviceability but also leads to large scale disintegration of the structure. A fracture criterion proposed by Hashin [3] and recently 
further developped to a practicable state by Puck [5] distinguishes between FF and IFF and therefore will be used in this study.

Another severe problem is the variability of loads and strength properties taking into account that such materials are used in highly optimized structures. Due to this optimisation and the fact, that many of the FRP used in practical applications show brittle fracture, laminated structures virtually are nonredundant, i. e. structural failure occurs as soon as local failure occurs.

As the stress distribution over the entire structure in general is not constant, the reliability analysis must be performed in multiple points of the structure. The stresses in laminated structures usually are calculated by Finite-Element-Analysis (FEA), which leads already to a discretization of the structure - the modelling of the structure by a mesh of finite elements. If it is then assumed that every finite element can be regarded as one failure element with a more or less constant stress state, the whole structure can be modelled as a series system of failure elements for the reliability analysis. However the entire structure's probability of failure must be independent from the number of elements in the FEA-mesh.

In the following a method will be presented that allows to calculate the probability of failure for structures showing series-system behaviour. The method will be applied to an example to test its efficiency.

\section{A multi-modal fracture criterion for unidirectionally fibre-reinforced plas- tics}

Unidirectionally fibre-reinforced plastics show two failure modes, fibre failure and matrix failure. Hashin proposed to use two independent criteria for FF and IFF [3]. He assumed that in the case of FF the fracture always occurs on a plane normal to the fibres. In case of IFF he postulated that a crack occurs parallel to the plane of maximum stresses in the matrix material and normal to the plane of layer isotropy (see figure 1). The stress $\sigma_{n n}$ acting normal to this plane provoques a mode-I crack of the matrix material. The longitudinal shear stress $\tau_{n 1}$ and the transverse shear stress $\tau_{n t}$ acting on this plane provoque mixed mode-II-mode-III failure.

He then proposed a simple fracture criterion for IFF where the stresses $\sigma_{n n}, \tau_{n 1}$ and $\tau_{n t}$ depend on the angle $\theta$ of the possible crackplane

$f\left(\sigma_{n n}, \tau_{n 1}, \tau_{n t}\right)=\left(\frac{\sigma_{n n}}{R_{n n}}\right)^{2}+\left(\frac{\tau_{n 1}}{R_{n 1}}\right)^{2}+\left(\frac{\tau_{n t}}{R_{n t}}\right)^{2} \leq 1$

$$
\begin{aligned}
{\sigma_{n n}}^{2} & =\left[\frac{1}{2}\left(\sigma_{22}+\sigma_{33}\right)+\frac{1}{2}\left(\sigma_{22}-\sigma_{33}\right) \cos 2 \theta+\tau_{23} \cdot \sin 2 \theta\right]^{2} \\
\tau_{n t}{ }^{2} & =\left[-\frac{1}{2}\left(\sigma_{22}-\sigma_{33}\right) \sin 2 \theta+\tau_{23} \cdot \cos 2 \theta\right]^{2} \\
\tau_{n 1}{ }^{2} & =\frac{1}{2}\left({\tau_{12}}^{2}+{\tau_{13}}^{2}\right)+\frac{1}{2}\left({\tau_{12}}^{2}-{\tau_{13}}^{2}\right) \cos 2 \theta+\tau_{12} \cdot \tau_{13} \cdot \sin 2 \theta
\end{aligned}
$$

The angle $\theta$ must be found by maximizing the left side of (1). Hashin considered the maximization of (1) as too expensive. Furthermore he did not see how to determine the 
compressive strength $R_{n n}^{c}$ for a mode I crack, which must be used in the first term of (1) for $R_{n n}$ in case of $\sigma_{n n} \leq 0$.

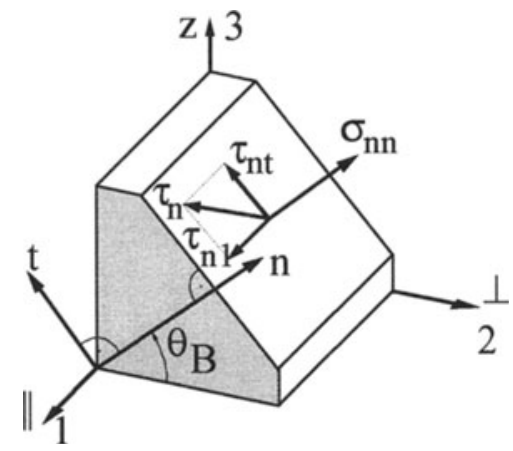

Figure 1: Definition of the coordinate systems for a lamina. The system 1-2-3 points with axis 1 (or II) in fibre direction, with axis 2 (or $\perp$ ) in transverse direction and with axis 3 (or z) through the thickness of the laminate.

Later Hahn, Tsai and Erikson showed, that the direction of a matrix crack in a unidirectional composite under combined loading can be determined by applying the energy release rate approach [2]. Assuming that the stresses $\sigma_{n n}, \tau_{n 1}$ and $\tau_{n t}$ cause mode-I, modeII and mode-III failure, respectively, they generated for the $\sigma_{22^{-}} \tau_{12}$-interaction a failure surface showing exactly the same shape and characteristics as Hashin's criterion (1), [5]. They also showed that for $\sigma_{n n} \leq 0$ the first term in (1) has to disappear as a mode-I crack does not open due to compression normal to the crack plane. Furthermore under pure compression $\sigma_{22}$ failure takes place as a mode-III crack under approximately $\pm 45^{\circ}$.

Finally Puck proposed some rules to determine the strengths required for (1) from standard tests [5]:

$R_{n n}^{t}=R_{22}^{t} \quad R_{n n}^{c} \rightarrow \infty \quad R_{n 1}=R_{12} \quad R_{n t}=\frac{R_{22}^{c}}{2}$

The index $i$ of a resistance quantity $R_{i j}$ specifies the plane parallel to the crack, the index $j$ is the direction of the stress acting on this plane (see figure 1). A superscript $t$ is used for tensile strength, the superscript $c$ for compressive strength, no superscript for shear strengths. The failure surface for combined loading $\sigma_{22}-\tau_{12}$ is shown in figure 3 as curve A with the associated angles of the crackplane $\theta$. 


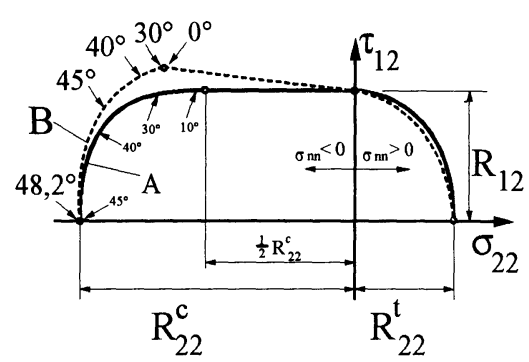

Figure 2: Fracture surfaces given by the Hashin-Puck criterion for the criterion (1) (curve A) and the improvement supposed by Puck (curve B).

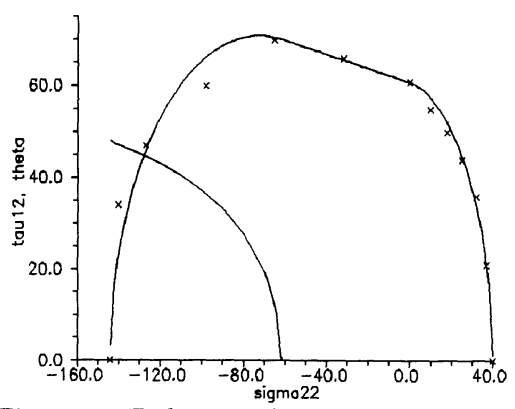

Figure 3: Failure surface and crack angle for criterion (3). Parameters are determined by fitting the curve to experimental data (crosses) with $\mu_{n 1}=\mu_{n t}=$ 0.15 for a typical GFRP [5].

Puck suggested the introduction of interaction terms into (1), leading to a fracture surface shown as curve B in figure $2[5]$. As compression on the crack surface certainly causes friction and therefore increases the shear strength, a possible modification of (1) is a criterion of the form

$$
\left(\frac{\max \left\{\sigma_{n n}, 0\right\}}{R_{n n}^{t}}\right)^{2}+\left(\frac{\tau_{n 1}}{R_{n 1}-\mu_{n 1} \cdot \sigma_{n n}}\right)^{2}+\left(\frac{\tau_{n t}}{R_{n t}-\mu_{n t} \cdot \sigma_{n n}}\right)^{2} \leq 1
$$

In case of $\sigma_{n n} \leq 0$ the parameters $\mu_{n 1}$ and $\mu_{n t}$ can be considered as coefficients of friction. Thus the parameter $\mu_{n t}$ can be determined from the crack angle $\Psi$ of a test specimen under transverse compression in analogy to the fracture hypothesis for soils by Mohr-Coulomb.

$\mu_{n t}=2 \Psi-\frac{\pi}{2} \quad R_{n t}=\frac{R_{22}^{c}}{2 \tan \Psi}$

The parameter $\mu_{n 1}$ must be determined by fitting (3) to results from tests with combined loading. The fracture surface for a typical glas fibre reinforced plastic (GFRP) under combined loading is shown in figure 3. In contrast to curve $\mathrm{B}$ in figure 2 the criterion (3) does not show a somewhat unreasonable kink where $\theta$ jumpes from $0^{\circ}$ to $30^{\circ}$. In this analysis the criterion (3) will be used for IFF. For FF the criterion proposed by Puck will be applied. A possible influence of shear stresses on $\mathrm{FF}$ is ignored.

$$
\left(\frac{\sigma_{11}}{R_{11}^{t}}\right)^{2} \leq 1 \quad \text { for } \sigma_{11} \geq 0 \text { and } \quad\left(\frac{\sigma_{11}}{R_{11}^{c}}\right)^{2} \leq 1 \quad \text { for } \sigma_{11} \leq 0
$$




\section{The size effect in series systems with variable number of components}

If the resistance of a brittle material is determined using a test specimen of volume $V_{0}$, which is much larger than the size of a single defect, it can be expected that the measured resistance includes the possibility of failure for all defects (with random size and orientation) in the test specimen. Also the local fluctuations of the stress state are incorporated in the specimens strength. Selecting the Weibull distribution as the natural stochastic model for the resistance of the material, the probability of failure of a structural element with volume $V_{1}$ under constant stress state $\sigma_{0}$ can be calculated by

$P_{f}\left(\sigma_{0}\right)=1-\exp \left[-\frac{V_{1}}{V_{0}}\left(\frac{\sigma_{0}-\tau}{w-\tau}\right)^{k}\right]$

The stress distribution of complicated structures can be calculated with finite-elementanalysis. As one basic assumption of this method is that a structure can be divided into numerous small elements for which a constant stress state can be assumed, formula (6) can be used as a model for the strength of one element. If it is assumed that, firstly, every element of the FEA-model of a structure is a failure element, secondly, the resistances of the individual elements are mutually independent, and, thirdly, the complete structure fails if one element fails, the structure's probability of failure can be calculated using the bounds by Ditlevsen [1] for series systems ( $F_{i}$ are the failure events):

$P_{f}=P\left(\bigcup_{i=1}^{n} F_{i}\right)=\left\{\begin{array}{l}\leq P\left(F_{1}\right)+\sum_{i=2}^{n}\left\{P\left(F_{i}\right)-\max _{j<i}\left\{P\left(F_{i} \cap F_{j}\right)\right\}\right\} \\ \geq P\left(F_{1}\right)+\sum_{i=2}^{n} \max \left\{0, P\left(F_{i}\right)-\sum_{j<i}^{n} P\left(F_{i} \cap F_{j}\right)\right\}\end{array}\right.$

For the reliability analysis of each element FORM methods [4] are used, giving $P_{f, i} \approx$ $\Phi\left(-\beta_{i}\right)$ where $\Phi(x)$ is the standard normal distribution and $\beta$ the safety index. The safety index is related to the $\beta$-point $\underline{u}^{*}$ by $\underline{u}^{*}=-\beta \cdot \underline{\alpha}$. As the problem is solved in standard normal space, the correlation coefficient $\rho_{i j}$ of two elements in the structure can be calculated by the scalar product $\rho_{i j}=\underline{\alpha}_{i}{ }^{T} \cdot \underline{\alpha}_{j}$. Thus the intersections in (7) take the correlation of the stress states in the individual elements into account.

\section{Implementation of the fracture criterion and the size effect into an existing software}

The fracture criterion (3) and the size-effect (6) were implemented into an existing software package for componential reliability analysis, COMREL [6]. In order to get the necessary information about the stress state in laminated structures, a three-dimensional anisotropic shell-plate-element - based on classical laminate theory and the Kirchhoff plate theory was added to the FEA-code NASCOM [7], which serves as preprocessor to the structural reliability software NASREL [8]. The finite elements so far have deterministic elasticity properties. Thus only the variability of the loads and of the local resistances are taken into account. The data flow is shown in figure 4 . 


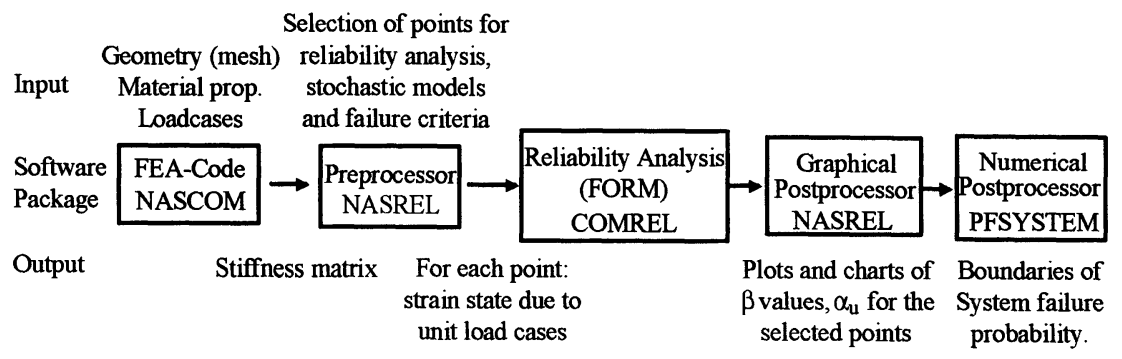

Figure 4: Integration of software packages and data flow for the calculation of the probability of failure of entire structures.

After the reliability analysis for each element of the structure plots of the distribution of $\beta$-values and $\alpha_{u}$-values allow a visual control of the results. Finally the probability of failure for the entire structure is evaluated using (7).

\section{Analysis of a strut under random loading}

The method as proposed above is illustrated at a strut. The geometry and the loads are shown in figure 5 , the stochastic model and the material properties of the carbon fibre reinforced plastic (CFRP) are given in figure 6. The stacking sequence of the laminate is $\left(90^{\circ} / 0^{\circ} / 0^{\circ} / 90^{\circ}\right)$ with $0^{\circ}$ pointing in the longitudinal direction of the strut, the thickness of the layers is $t_{i}=(0.15 / 1.35 / 1.35 / 0.15)$ (all in [mm]).

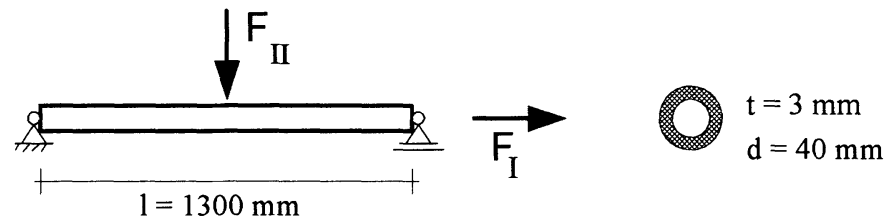

Figure 5: Example: a strut under longitudinal tension $F_{I}$, a bending force $F_{I I}$ in the middle and constant temperature $\Delta T=-100^{\circ} \mathrm{C}$.

One half of the strut is divided into 16 elements in radial direction and into 13 elements in longitudinal direction (208 in total). As a second case, the strut is divided into 26 elements in longitudinal direction (416 in total). To demonstrate the effect of the Weibullsize-effect the structure is analyzed with the two different meshes. The reliability analysis has to be performed at each boundary of a layer for every element and both FF and IFF must be considered. As an example the influence of the Weibull size effect on the probability of failure is demonstrated for one layer boundary. The results for the outer boundary of the $0^{\circ}$-layer are presented in figure 7 . The first column shows the number of elements of the FEA-model for the strut, the second indicates whether the Weibull-scaling 


\begin{tabular}{ccc}
\hline Variable & Mean value & C.o.V. \\
\hline$E_{11}$ & 170000 & - \\
$E_{22}$ & 9000 & - \\
$\nu_{12}$ & 0.28 & - \\
$\alpha_{T, 1}$ & $-3.0 \cdot 10^{-6}$ & - \\
$\alpha_{T, 2}$ & $4.0 \cdot 10^{-5}$ & - \\
$R_{11}^{t}$ & 2900 & 0.04 \\
$R_{11}^{c}$ & 1620 & 0.08 \\
$R_{22}^{t}$ & 60 & 0.10 \\
$R_{22}^{c}$ & 165 & 0.09 \\
$R_{12}$ & 86 & 0.04 \\
\hline
\end{tabular}

\begin{tabular}{cccc}
\hline Variable & Mean value & C.o.V. & model \\
\hline Tension & 209000 & 0.1 & Lognormal \\
Bending & 3600 & 0.1 & Lognormal \\
Temperature & -100 & 0.05 & Lognormal \\
$R_{11}^{t}$ & 2900 & 0.04 & Weibull \\
$R_{11}^{c}$ & 1620 & 0.08 & Weibull \\
$R_{n n}^{t}$ & 60 & 0.10 & Weibull \\
$R_{n 1}$ & 186 & 0.04 & Weibull \\
$R_{n t}$ & 71 & 0.1 & Weibull \\
$\mu_{n 1}$ & 0.15 & 0.1 & Lognormal \\
$\mu_{n t}$ & 0.15 & 0.1 & Lognormal \\
\hline
\end{tabular}

Figure 6: Mechanical properties of CFRP used for the example (left table) and set of basic variables for the reliability analysis of the strut (right table).

according to (6) has been taken into account. The third column contains the number of failure components for the series system, the fourth shows the bounds of $\beta$, and the last column gives the upper bound of system- $P_{f}$. It should be mentioned that the intersection probabilities in (7) of at most the first 800 larger probability events had to be computed in order to obtain the rather narrow reliability bounds.

\begin{tabular}{ccccc}
\hline Meshsize (FEA) & Scaling & Failure components & range of $\beta$ & $P_{f}$ \\
\hline 208 & yes & 3328 & $2.81 \leq \beta \leq 2.81$ & $\leq 0.0025$ \\
416 & yes & 6656 & $2.81 \leq \beta \leq 2.81$ & $\leq 0.0025$ \\
208 & no & 3328 & $-0.22 \leq \beta \leq 0.52$ & $\leq 0.5854$ \\
416 & no & 6656 & $-36.62 \leq \beta \leq 0.40$ & $\leq 1.0000$ \\
\hline
\end{tabular}

Figure 7: Probability of failure for one layer boundary, including both FF and IFF.

It can be seen, that ignoring the scaling of resistances produces severe errors. For completeness the probability of failure has been evaluated for the entire strut. For 208 (416) elements having $4 \beta$-points each (due to the geometrical model of the shell-plateelement), 8 layer boundaries and two criteria the number of failure components in the series system is 19968 (39936). The results are given in figure 8 .

\section{Conclusions}

It has been shown that modern FORM methods can be coupled with finite element codes for the analysis of laminated structures involving a large number of failure modes. The strength criteria proposed by Hashin/Puck for both fibre and interfibre failure have been used. It can be demonstrated that the use of some other classical criterion can lead to 


\begin{tabular}{ccc}
\hline Meshsize (FEA) & 208 & 416 \\
Failure components & 19998 & 39936 \\
Componential $\beta$ & $4.34 \leq \beta \leq 10.96$ & $4.40 \leq \beta \leq 11.13$ \\
System bounds (all comp.) & $2.33 \leq \beta \leq 2.34$ & $2.34 \leq \beta \leq 2.34$ \\
System prob. of failure & $0.0096 \leq P_{f} \leq 0.0098$ & $0.0096 \leq P_{f} \leq 0.0096$ \\
\hline
\end{tabular}

Figure 8: Results for the entire strut, including both FF and IFF.

inappropriately designed structures. The outspoken size effect must be taken into account correctly both within the finite element and across the elements. The systems reliability can be narrowly bounded even for a large number of failure modes.

\section{References}

1. O. Ditlevsen, "Narrow Reliability Bounds for Structural Systems", Journal of Structural Mechanics, Vol. 7, 435 - 451, (1979)

2. H. T. Hahn, J. B. Erikson and S. W. Tsai, "Characterization of Matrix/InterfaceControlled Strength of Unidirectional Composites", Fracture of Composite Materials, Martius Nijhoff Publ., Den Haag, 197 - 214, (1982)

3. Z. Hashin, "Failure Criteria for Unidirectional Fibre Composites", Journal of Applied Mechanics, Vol. 47, 329 - 334, (1980)

4. M. Hohenbichler, S. Gollwitzer, W. Kruse and R. Rackwitz, "New Light on Firstand Second-Order Reliability Methods", Structural Safety, Vol. 4, Elsevier Science Publishers B. V., 267 -284, (1987)

5. A. Puck, "Ein Bruchkriterium gibt die Richtung an", Kunststoffe, 82. Jahrgang, 607 - 610, (1992)

6. COMREL, Software for componential reliability analysis, RCP GmbH, München, Germany,(1994)

7. NASCOM, Software for finite element analysis, RCP GmbH, München, Germany, (1994)

8. NASREL, Software for reliability analysis of structures modelled by finite elements, RCP GmbH, München, Germany, (1994) 